\title{
Using Business Process Management Notation to Model Therapeutic Prescription Protocols: The PrescIT Approach
}

\author{
Pantelis NATSIAVAS ${ }^{\mathrm{a}, 1}$, Thanos G. STAVROPOULOS ${ }^{\mathrm{b}}$, Antonios PLIATSIOS ${ }^{\mathrm{b}}$, \\ Haralampos KARANIKAS ${ }^{\mathrm{c}, \mathrm{d}}$, George I. GAVRIILIDIS ${ }^{\mathrm{a}}$, Vlasios K. DIMITRIADIS ${ }^{\mathrm{a}}$, \\ George NIKOLAIDIS ${ }^{\mathrm{e}}$, Spiros NIKOLOPOULOS ${ }^{\mathrm{b}}$, Petros SKAPINAKIS ${ }^{\mathrm{f}}$, \\ Eleytherios THIREOS ${ }^{\mathrm{c}, \mathrm{d}}$ and Ioannis KOMPATSIARIS ${ }^{\mathrm{b}}$ \\ a Institute of Applied Biosciences, Centre for Research \& Technology Hellas, \\ Thessaloniki, Greece \\ ${ }^{\mathrm{b}}$ Information Technologies Institute, Centre for Research \& Technology Hellas, \\ Thessaloniki, Greece \\ ${ }^{\mathrm{c}}$ Department of Computer Science and Biomedical Informatics, University of Thessaly, \\ Lamia, Greece \\ d Athens Medical Society, Athens, Greece \\ ${ }^{\mathrm{e}}$ ERGOBYTE S.A., Thessaloniki, Greece \\ ${ }^{\mathrm{f}}$ Faculty of Medicine, University of Ioannina, Ioannina, Greece
}

\begin{abstract}
Clinical Decision Support Systems (CDSS) could play a prominent role in preventing Adverse Drug Reactions (ADRs) especially when integrated in larger healthcare systems (e.g. Electronic Health Record - EHR systems, Hospital Management Systems - HMS, e-Prescription systems etc.). This poster presents an approach to model Therapeutic Prescription Protocols (TPPs) via the Business Process Management Notation (BPMN), as part of the e-Prescription CDSS developed in the context of the PrescIT project.
\end{abstract}

Keywords. Adverse Drug Reactions, Drug Safety, Clinical Decision Support Systems, e-Prescription, Business Process Management Notation

\section{Introduction}

Adverse Drug Reactions (ADRs) are a major public health issue of large financial impact, causing a huge number of hospitalizations and even deaths [1]. Advances in Information and Communication Technologies (ICT) enable the use of new technological paradigms to support drug safety within the clinical environment and beyond. The PrescIT project ${ }^{2}$ aims to develop a CDSS platform to support safe e-Prescription, using a rule-based approach. To this end, it aims to leverage the recently developed Therapeutic Prescription Protocols (TPPs), which formalize general, widely acceptable Clinical

\footnotetext{
1 Corresponding Author, Pantelis Natsiavas, Institute of Applied Biosciences, Centre for Research \& Technology Hellas, 6th Km. Charilaou - Thermi Rd, GR57001, Thessaloniki, Greece; E-mail: pnatsiavas@certh.gr

${ }^{2}$ The PrescIT project: https://www.prescit.com/
} 
Practice Guidelines (CPGs) [2]. Specifically, TPPs were developed initially by the Greek National Organization for Medicines' (EOF) in free text form and converted in algorithmic form during the project "Medicines Prescription eProtocols", to be integrated in the Greek national e-Prescription system, by the Athens Medical Society ${ }^{3}$ (AMS).

\section{Methods}

We investigated the use of Business Process Management Notation $(\mathrm{BPMN})^{4}$ as the main formalism to represent TPPs. A proof-of-concept TPP for depression was encoded in BPMN and implemented in $\mathrm{jBPM}^{5}$, which aims to select one of three depression "stages" corresponding to different prescription medication for treatment.

\section{Results and Discussion}

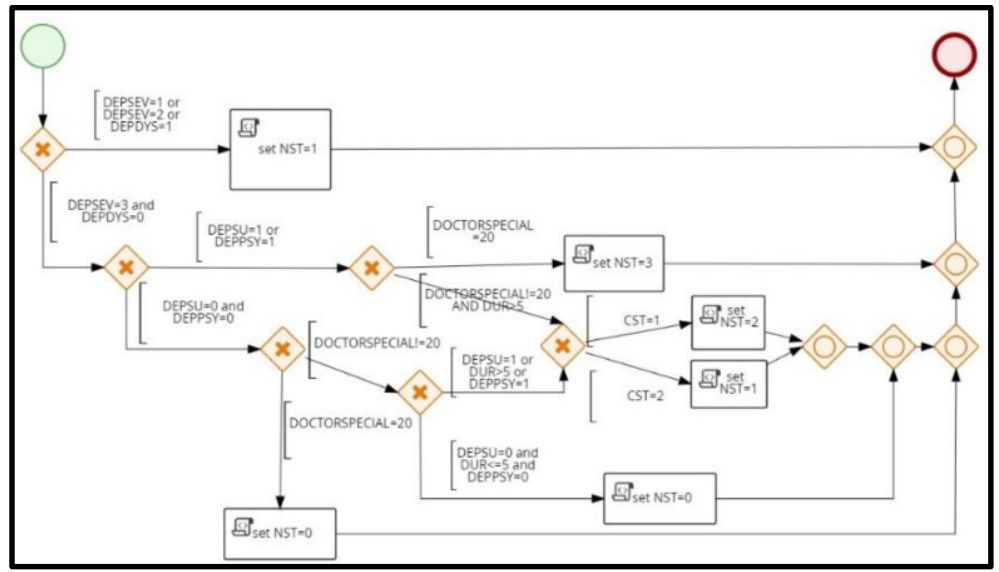

Figure 1. The BPMN model of the TPP for depression in jBPM

The implemented TPP in BPMN produces the desired output and visualizes the process (Figure 1). Based on this experience, BPMN, although not tailored for medical scenarios, provides significant benefits (e.g. in terms of visualization, understanding and execution).

\section{References}

[1] Formica D, Sultana J, Cutroneo PM, Lucchesi S, Angelica R, and Crisafulli S, The economic burden of preventable adverse drug reactions : a systematic review of observational studies, Taylor \& Francis, 2018. doi:10.1080/14740338.2018.1491547.

[2] Karanikas H, Papadakis M, and Thireos E, Development Of Prescription E-Protocols For Medicines And Integration On The Greek National E-Prescription System, Value Heal. 2015;18:A385. doi:10.1016/j.jval.2015.09.835.

\footnotetext{
${ }^{3}$ http://www.mednet.gr

4 https://www.bpmn.org/

5 https://www.jbpm.org/
} 